\title{
REFLEXIONES CURRICULARES: UNA CONVERSACIÓN SOBRE NUESTRA EXPERIENCIA EDUCACIONAL
}

\section{CURRICULAR REFLECTIONS: A CONVERSATION ABOUT OUR EDUCATIONAL EXPERIENCE}

Daniel F. Johnson Mardones

$P h D$

Department of Curriculum and Instruction

University of Illinois Urbana Champaign

1310 S. 6th St., Champaign, Illinois 61820

dfjohns2@illinois.edu

Resumen: Este texto reflexiona acerca de la naturaleza del campo curricular en tanto estudios de la experiencia educacional. Lo hace utilizando la metáfora de la conversación. Se mueve entonces entre la voz académica y la biográfica personal volviéndose sobre la experiencia educacional del autor en relación con los estudios curriculares. Desde dicha experiencia se abordan tres temas que parecen relevantes para aquellos que dedican sus esfuerzos al estudio y la enseñanza del curriculum. Estos temas son: internacionalización, estandarización, y el estudio de los estudios curriculares.

Palabras clave: Curriculum, estudios curriculares, internacionalización, experiencia educacional, estandarización.

Abstract: This text reflects about the nature of the curriculum field as study of educational experience. In doing so, it uses the metaphor of conversation. The text moves between the academic voice and the personal voice tuning onward the author's educational experience regarding curriculum studies. From that experience, it addresses three relevant themes that seem to be relevant for those who focus their efforts to study and teach curriculum. These themes are internationalization, standardization, and the study of curriculum studies. 
Keywords: Curriculum, curriculum Studies, internationalization, educational experience, standardization

Una reflexión curricular es una conversación sobre la experiencia educacional. Una conversación complicada (Pinar 2012, 2008, 2000[1975]; Goodson, 2011). Esta complicación se debe en primer lugar a la naturaleza compleja del fenómeno curricular. Esta complejidad se debe en gran medida a la multiplicidad de usos de para la palabra curriculum. En Efecto el uso de calificativos antepuestos al sustantivo curriculum ha generado un creciente número de tipos de curricula (Glatthorn, 2006; Johnson-Mardones, 2014; Schubert, 2008). Cada uno de estos curricula enfatiza una dimensión de curriculum como fenómeno. De esta manera, encontramos un curriculum oficial, explicito, escrito, apoyado, en acción, implementado, enseñado, implementado, evaluado, implícito, oculto, nulo, aprendido, paralelo, etc.; dependiendo de la múltiples clasificaciones desarrolladas en la historia del campo curricular, mayoritariamente en los Estados Unidos (Eisner, 1979; Goodlad, 1979; Grundy, 1987). Curriculum es entonces una conversación sobre un fenómeno complejo, un fenómeno multidimensional. Curriculum es un esfuerzo académico por entender dicho fenómeno, dicha complejidad.

Esta conversación es también complicada cuando miremos el curriculum desde el punto de vista de quien experimenta el contenido de la escolaridad: el estudiante. En otras palabras, cuando entendemos curriculum como lo vivido, como la experiencia educacional vivida. Curriculum entonces se convierte en una reflexión sobre la vivencia interna de las experiencias externas, para usar la expresión de Sartre (1963). Es en esa vivencia, que todos vivimos de manera personal, que nos relacionamos individual y colectivamente con cuerpos de conocimientos organizados como disciplinas escolares y académicas. Para mi esos cuerpos de conocimiento han sido la historia, y de manera más amplia las ciencias sociales y las humanidades; y, en educación los estudios curriculares y la didáctica, particularmente la didáctica de la historia y las ciencias sociales.

Teniendo estas primeras reflexiones como punto de partida, me centrare a continuación en mi experiencia educacional con aquel campo que estudia la experiencia educacional, es decir, los estudios curriculares. 
En otras palabras, me centrare en mi curriculum como currere, la raíz latina de la palabra curriculum que enfatiza la acción de recorrer el curso de estudios, el curriculum vivido (Pinar, 2000[1975]). En cierto modo, esa es la forma en que siempre entendí la palabra curriculum. Primero obviamente en relación con curriculum vitae, y luego en relación con el programa de estudio de un curso y el contenido de una clase en mi programa de formación inicial de profesores. Siempre pensé que lo más importante era lo que sucedía en la sala de clases más allá de la importancia de cualquier prescripción o planificación de la enseñanza. La conceptualización por supuesto vino después. Con todo, creo importante decir que tal vez fue esa distancia que veía en un concepto de curriculum, todavía definido desde lo que ahora llamamos un enfoque tecnocrático, lo que me llevó a la didáctica específica de la historia y las ciencias sociales como primer campo de ocupación dentro del campo educacional. Al menos en el campo de la didáctica específica había una mayor cercanía al contenido y práctica de la enseñanza. Entonces curriculum parecía una formalización extrema. Un "entonces" que sucedió alrededor de 3 décadas después de que esa perspectiva entrara en crisis en su contexto de origen; y, desde que las primeras voces latinoamericanas criticaron el arribo y acrítica aplicación de esa "nueva" tecnología educacional en nuestro contexto.

Esa desconfianza forjada en mis tiempos de estudiante se confirmó en mis primeras experiencias docentes casi contemporáneas con mi participación en procesos de formación docente, inicial y permanente. Desde esta disconformidad nació una búsqueda por una reflexión teórica que diera cuenta de la complejidad del fenómeno educativo. Desde la posición ventajosa del presente puedo decir que se trataba de la búsqueda de un discurso educativo dentro del campo educacional. Esa búsqueda me llevo a mi concepción de curriculum como curriculum vivido; $y$, consecuencialmente, a las historias de vida y estudios biográficos y narrativos en educación y formación de profesores. En estos últimos años, curriculum como currere ha ocupado un lugar central en mis reflexiones en torno a la experiencia educacional y a la aproximación metodológica para entenderla.

\section{CURRICULUM COMO CURRERE}

Mi participación en la implementación de la reforma curricular en educación básica y media me había ya devuelto al campo curricular 
que ahora se mostraba con mayor pluralidad discursiva. De esta manera, en los primeros años del siglo XXI la multiplicidad de enfoques en curriculum era ya parte de formación académica y profesional. También era consciente de la separación entre teoría y diseño curricular, aunque aún no se convertía en una preocupación fundamental en mi quehacer. Fue en este escenario donde comencé a encontrar textos que se acercaban a mi intuitiva concepción de curriculum como curriculum vivido; esto es curriculum como currere. En otras palabras, se trata de dejar de considerar la definición de curriculum en su forma sustantiva, estática, por tanto, de curso de estudio y pasar a pensarla como curso de vida, como currere, la forma verbal, por lo mismo dinámica, del sustantivo curriculum.

Mi primer encuentro explicito con la palabra currere tuvo lugar durante mis estudios en el programa de Magister en Educación con mención en Curriculum y Comunidad Educativa, en la Universidad de Chile. En esos tiempos me encontraba en la búsqueda de un concepto que me permitiera conectar la teoría curricular y formación de profesores. El contexto de esa búsqueda: mi tesis de magister. Una cita en un artículo leído en el seminario de teoría curricular me habida cautivado: "no hay mejor manera que estudiar curriculum que estudiarnos nosotros mismos." (Connelly y Clandinin, citado in Pinar, 2008: 498). Siguiendo referencias, encontré un artículo leído a comienzos de los setenta en el encuentro anual de la American Educational Research Association (AERA) titulado The Method of Currere, el método currere. El autor William Pinar definía ahí curriculum como una complicada conversación sobre la experiencia educacional de cada uno... El autor usaba la misma expresión currere para referirse al método que permitía conocer esa experiencia educacional. Era un concepto curricular forjado para pensar la experiencia educacional de los profesores en formación. Había encontrado el concepto que me permitiría conectar teoría curricular y formación de profesores. Otro efecto de ese encuentro fue mi deseo de profundizar mi estudio de los estudios curriculares.

El uso específico del concepto currere, curriculum como curriculum vivido, me sirvió para entender la formación docente y la identidad profesional como una narrativa que proporcionaba cierta coherencia a la historia de vida integrando la biografía personal, la experiencia de formación inicial, y la socialización profesional. Fue la fenomenología sociológica, particularmente la estructura del mundo de 
vida delineada por Schütz, el lebenswelt (Schütz, 1974; Schütz y Luckmann, 1973), la que me permitió situar la identidad docente en el eje temporal que va desde el reino de los antecesores y los predecesores. Este eje temporal, eje al que Schütz no presto demasiada atención, fue desarrollado por sus discípulos Berger y Luckmann en la construcción social de la realidad (2001). Al final de mi formación de magister escribía:

Currere al igual que curriculum estaba referido al concepto mismo de curriculum como experiencia educacional, y también al método por el cual se accedía a dicha experiencia. Se trata de reconstruir narrativamente el curriculum experimentado por cada uno de estos profesores de historia principiantes para convertirse en los profesores que hoy día son. Cada una de estas historias ha sido, por lo tanto, leída como su currere; esto es, leída educacionalmente como un intento de llevar adelante esta complicada conversación acerca de su experiencia educacional. (Johnson-Mardones, 2010, p. 10).

Esta conexión con la fenomenología estuvo ya presente en el nacimiento del concepto y método de currere (junto con el existencialismo y el psicoanálisis), como aprendería más tarde. No esta demás decir que junto con el haber encontrado la conexión entre la teoría curricular y la formación de profesores que necesitaba, un tercer campo se conectaba a esta reflexión: el de las metodologías cualitativas. Esa parte de la historia la dejare para otro momento.

\section{ESTUDIOS CURRICULARES: ALGUNOS TEMAS RELEVANTES}

Con el contexto que provee esta historia, mi historia, pero también la historia del campo curricular; me atrevo a plantear algunos temas relevantes para este discurso académico educativo acerca de la educación que llamamos estudios curriculares.

\section{Internacionalización}

El campo académico de los estudios curriculares ha estado históricamente informado por la idea de una cultura nacional (Da Silva, 1999, Pinar, 2014a). El estado-nación ha sido la unidad de prescripción 
y análisis en el campo curricular. Esto es todo, pero extraño. En cuanto fenómeno histórico, como contenido de la escolaridad, el curriculum surge como aquella herramienta que permite y controla la transmisión de la herencia cultural en la sociedad moderna. Para ser más preciso, el currículum nace como un dispositivo homogeneizador y estandarizador de la diversidad cultural presente dentro de los límites nacionales modernos. (Johnson-Mardones, 2015a, p. 8).

En efecto, el moderno uso de la palabra currículum está ligado al surgimiento del sistema escolar moderno, es decir, la escuela. La modernidad políticamente triunfante en las revoluciones liberales de los siglos XVIII y XIX creó los sistemas escolares modernos. Estos fueron concebidos como sistemas constructores de nación, una tarea principal para recientemente triunfante Estado moderno. Así, la escuela [y el curriculum] será la máquina destinada a producir una comunidad imaginada, la nación; para usar la asertiva expresión acuñada por Anderson. (Johnson-Mardones, 2015a, p. 7)

Teniendo aun lo nacional como unidad de prescripción y análisis, el campo curricular se ha visto afectado por las transformaciones del fin de siglo pasado. De esta manera, durante las últimas décadas hemos presenciado un interés creciente por estudiar y entender curriculum de manera internacional (Autio, 2006; Pinar, 2003, Pinar et. al., 1995; 2014a, 2014b; Popkewitz, 2013; Ropo \& Autio, 2009; Westbury et al., 2000). El concepto de internacionalización, entonces, se relaciona con el esfuerzo emprendido en el cambio de siglo por diversos académicos de distintos países de desarrollar una comprensión verdaderamente internacional del campo curricular. En este sentido, internacionalización desafía el quehacer de quienes se dedican a la enseñanza e investigación en estudios curriculares.

Lo que hoy se conoce como internacionalización en curriculum se ha institucionalizado en 5 conferencias trianuales sostenidas desde 2001, año de la fundación de la International Association for the Advancement of Curriculum Studies (IAACS). La última de estas conferencias fue celebrada en mayo 2015 en Ottawa, Canadá. Junto con su institucionalización, el movimiento de internacionalización del campo curricular se ha hecho público con la publicación de las dos versiones del International Handbook of Curriculum Research (Pinar ed., 2003, 2014b). Otra presencia institucional pública del movimiento de 
internacionalización lo constituye la revista de la IAACS: Transnational Curriculum Inquiry.

Con todo, si miramos a lo internacional desde el sur global (De Sousa Santos, 2014) podemos darnos cuenta de que internacionalización ha sido una dimensión de este campo académico por lo menos desde los años sesenta. En efecto, el concepto de curriculum que prevalece hoy globalmente es uno heredado de los tiempos en que el campo era dominado por la perspectiva del desarrollo curricular. Dicha perspectiva fue exportada globalmente durante la década de los setenta junto con el concepto de planificación educacional que informo las reformas educacionales de ese entonces. Dicho proceso significo la aplicación mecánica de conceptos y procedimientos traídos desde el centro sin ninguna consideración por los contextos locales. Los estudios curriculares arribaron a América Latina durante esta primera ola de internacionalización más en la forma de un monologo cultural que de un dialogo intercultural. Los efectos de esa historia esperan todavía ser contados. De esta manera, esta primera internacionalización reforzó dinámicas neocoloniales cuyo revestimiento estético apelaba a un saber tecnológico propio de lo que hoy llamamos racionalidad instrumental. Latinoamérica sigue siendo un valioso y necesario recordatorio de que la internacionalización ha sido parte del campo curricular desde la consolidación de ese campo en el contexto anglo-norteamericano.

\section{Estandarización}

Es por supuesto la globalización y movilidad internacional, sus procesos y debates, el fenómeno que ha dado impulso a la internacionalización de los estudios curriculares. Esto nos lleva a un segundo punto: estandarización. Esta es un fenómeno propio de la escolaridad y el curriculum es en efecto su herramienta principal desde el surgimiento de la escuela como institución moderna. Esta idea se expresó también en el arribo de los estudios curriculares a nuestra región. Hoy, sin embargo, esta característica de los sistemas educativos se ha acentuado hasta el punto de que amenaza con hacerse sinónimo de educación.

La idea de que una tecnología educativa, la planificación educacional y el desarrollo curricular, solucionarían los 
problemas de la región reemplazaban entonces los contenidos de la "cultura europea" de la educación decimonónica. La lógica, sin embargo, de que una solución externa debe ser aplicada sin consideración de los contextos particulares permanece. De alguna forma, esta visión es el anticipo y continuación de la idea de una cultura global, tecnológica, donde las relaciones culturales son redefinidas en términos de la articulación local-nacional-global. (Johnson-Mardones, 2015a, p. 9)

En dicho contexto, educación pareciera ser no solo estandarización sino más aún estandarización reducida la evaluación estandarizada. Esta exacerbación de la estandarización amenaza aquellas caracterizas emancipadoras que desde sus comienzos fueron parte del sistema escolar. En términos curriculares es posible sostener que un curriculum global está siendo impuesto cada vez con mayor fuerza a través de la implementación de evaluaciones estandarizadas a nivel internacional. En este sentido, una reflexión curricular internacional, global si se quiere, no es solo deseable sino indispensable a nivel nacional. Y esto dado que las evaluaciones nacionales tienden a alinearse a las internacionales. De esta forma, el curriculum evaluado como principal dimensión del fenómeno curricular hoy a nivel nacional, que dado lo dicho es ya global. Tal vez, lo más preocupante de este fenómeno es que esta implementación se ha dado casi sin discusión. La estandarización cierra los espacios de conversación y dialogo, particularmente de un dialogo intercultural tan necesario en los tiempos que vivimos. Por lo menos en el modelo de Tyler había cierto nivel de discusión al nivel de los objetivos. Hoy la selección cultural es crecientemente desplazada de los centros de nacionales hacia el nivel global.

\section{El Estudio de Los Estudios Curriculares}

El último tema que quiero destacar aquí como relevante para los estudios del curriculum es el estudio de los estudios curriculares. Primero porque los de los estudios curriculares son un campo dedicado a entender el fenómeno curricular, y nuestro entender es generalmente a un proceso de estudio. Para entender el fenómeno curricular es necesario 
estudiar la experiencia educacional: la nuestra, la de nuestros antepasados, la de nuestros sucesores. Para entender el campo curricular es también necesario estudiar el esfuerzo académico dedicado a este estudio. Los estudios curriculares nos ofrecen un discurso educacional sobre la educación. Su sofisticación actual presenta un potencial más allá del campo educacional mismo.

Así, en segundo lugar, es necesario el estudio del campo curricular a nivel internacional, pero particularmente en su contextualización y desarrollos autónomos a nivel nacional y local. Este estudio debe ser obviamente bidimensional a lo menos. Esto es, histórico y conceptual. En términos concretos el estudio de los 50 años de historia del campo curricular en Chile es urgente. En este sentido Mario Leyton (2004) ha sostenido que junto a la historia del campo en los Estados Unidos debiera sumarse la reflexión de la historia del curriculum en Chile.

Este estudio requiere ser situado en relación con la historia del campo en Latinoamérica e internacional. Esta historia intelectual debe ser también historia del presente, permitiendo conectar los conceptos centrales usados para entender la experiencia educacional hoy con su desarrollo histórico. Estamos hablando, entonces, del estudio de la producción intelectual del campo curricular, por limitada que esta sea. Estamos hablando, también, de estudio que debe hacerse hecho público para incrementar el conocimiento curricular disponible para pensar el pasado, presente y futuro de la educación. Así definida, el tema del estudio no solo fundamental para los estudios curriculares sino para nuestras sociedades en general; y particularmente para la formación del profesorado.

\section{CONCLUSIÓN}

Al comienzo de la segunda década del siglo XXI, los estudios curriculares han sido definidos como un campo divergente moviéndose en direcciones diversas (Pinar, 2011). Personalmente pienso que esa es la debilidad y la fortaleza de este campo. Estas tendencias centrípetas se explican por la historia de este campo, particularmente por la historia de las últimas décadas del siglo pasado, y en especial por la renovación intelectual del campo en el contexto anglo-norteamericano conocida como reconceptualización. El paisaje intelectual que emergió de este 
proceso ya no era teorético, crecía en su comprensión histórica. Ciertamente, esta renovación intelectual debe ser hoy profundizada en perspectiva internacional. En nuestro caso específico, esa renovación necesita ser puesta en relación con la producción intelectual en nuestro contexto nacional y latinoamericano. Este último, requiere, además, una creciente y consistente esfuerzo por integrar nuestra tradición intelectual en la enseñanza de los estudios curriculares a nivel de pregrado y posgrado.

Nota:

El presente trabajo es una versión ampliada y editada de la presentación realizada por el autor en el Seminario Permanente del Magister en Educación con mención en Currículo y Comunidad Educativa del Departamento de Educación, Facultad de Ciencias Sociales, Universidad de Chile en octubre de 2014.

\section{REFERENCIAS BIBLIOGRÁFICAS}

Autio, T., 2006

Subjectivity, curriculum and society. Mahwah, NJ: Erlbaum.

Berger, P. y Luckmann, La construcción social de la T., 2001 realidad. Amorrortu: Buenos Aires.

Da Silva, T., 1999

De Souza, B., 2014

Goodlad, J., 1979

Goodson, I., 2011

Grundy, S., 1987
Documentos de identidade. Belo Horizonte: Autentica.

Epistemologies of the South. London: Paradigm.

Curriculum inquiry: The study of curriculum practice. New York, NY: McGraw-Hill.

Life politics conversations about culture and education. Rotterdam: Sense.

Curriculum: product or praxis. London: Falmer Press. 
Johnson, D., 2016

Johnson, D., 2015a

Johnson, D., 2014

Johnson, D., 2010

Leyton, M., 2005

Miller, J., 2009

Pinar, W., 2014a

Pinar, W. (Ed.), 2014b

Pinar, W., 2008
Editorial. Investigación Cualitativa, 1(1), 1-5.

Formar ciudadanos interculturales en un mundo global: Algunas Notas desde los estudios curriculares. Diálogo Andino. 47(2) 7-14.

Toward a multidimensional concept of Curriculum: Understating curriculum as phenomenon, field and design. European Journal of Curriculum Studies (Euro-JCS), 1 (2) 72-77.

Identidad y formación docente de los profesores principiantes. Un enfoque biográfico-narrativo. Congreso Iberoamericano de Educación. Septiembre 14- 16, Buenos Aires.

El Curriculum como disciplina y su desarrollo. En V. Soto and O. Reveco (Eds.). Problemáticas del curriculum nacional hoy. Santiago: Arcis.

Curriculum studies and transnational flows and motilities. En E. Ropo y T. Autio (Eds.), (s/f) International conversations on curriculum studies. Rotterdam: Sense.

La teoría del curriculum. Narcea: México.

International handbook of curriculum research. Mahwah, NJ: Erlbaum.

Curriculum theory since 1950: Crisis, reconceptualization, internationalization. En M. Connelly, M. F. He, and J. Phillion (Eds.). The SAGE handbook of 
Pinar, W., 2006

Pinar, W. (Ed.), 2003

Pinar, W., 2000 [1975] curriculum and instruction. Los Angeles, CA: Sage Publications.

The Synoptic Text Today and other essays: Curriculum Development after then Reconceptualization. New York, NY: Peter Lang.

International handbook of curriculum research. Mahwah, NJ: Erlbaum.

Currere: toward reconceptualization. Pinar, W. (Ed.) Curriculum theorizing: The Reconceptualization. Troy, NY: Educator's International Press.

Pinar, W., Reynolds, W., Understanding curriculum: An Slattery, P., Taubman, P., introduction to historical and 1995

Popkewitz, T., 2013

Ropo E. \& Autio, T., 2009 International conversations on curriculum studies. Rotterdam: Sense.

Sartre, J., 1963

Schütz, A., 1974

Rethinking the history of education. New York, NY: Palgrave Macmillan. contemporary curriculum discourses. New York: Peter Lang.

Search for a method. New York, NY: Knopf.

Estudios sobre teoría social. Amorrortu: Buenos Aires. 1974. Schütz, A. y Luckmann, T. (1973). Las estructuras del mundo de la vida. Amorrortu: Buenos Aires.

Westbury, I., Hopmann, Teaching as a reflective practice: S., \& Ri- quarts, K. (Eds.), The German Didaktik tradition. 2000
Mahwah, NJ: Erlbaum. 